\title{
Ratiometric, Filter-free Optical Sensor Based on a Complementary Metal Oxide Semiconductor Buried Double Junction Photodiode
}

Ka Yi Yung ${ }^{1, \mathrm{a}}$, Zhiyong Zhan ${ }^{2, \mathrm{a}}$, Albert H. Titus ${ }^{2}$, Gary A. Baker ${ }^{3}$ and Frank V. Bright ${ }^{*}$

a) Authors contributed equally to this work. *Corresponding author (chefvb@buffalo.edu)

\author{
${ }^{1}$ Department of Chemistry and ${ }^{2}$ Department of Biomedical Engineering \\ University at Buffalo, The State University of New York, Buffalo, NY 14260 \\ ${ }^{3}$ Department of Chemistry, University of Missouri, Columbia, MO 65211-7600
}

\begin{abstract}
We report a complementary metal oxide semiconductor integrated circuit (CMOS IC) with a buried double junction (BDJ) photodiode that (i) provides a real-time output signal that is related to the intensity ratio at two emission wavelengths and (ii) simultaneously eliminates the need for an optical filter to block Rayleigh scatter. We demonstrate the BDJ platform performance for gaseous $\mathrm{NH}_{3}$ and aqueous $\mathrm{pH}$ detection. We also compare the BDJ performance to parallel results obtained by using a slew scanned fluorimeter (SSF). The BDJ results are functionally equivalent to the SSF results without the need for any wavelength filtering or monochromators and the BDJ platform is not prone to errors associated with source intensity fluctuations or sensor signal drift.
\end{abstract}

Key words: Buried double junction, complementary metal oxide semiconductor, integrated circuit, fluorescence, ionic liquid, sensing. 
Abbreviation: BDJ - buried double junction; CMOS IC - complementary metal oxide semiconductor integrated circuit; SSF - slew scan fluorimeter; $\left(\left[\mathrm{P}_{14,666}^{+}\right]\right)^{-}$ trihexyl(tetradecyl)phosphonium cation; $\mathrm{HPTS}^{3-}$ - 8-hydroxypyrene-1,3,6-trisulfonate; SNARF1 - seminaphthorhodamine-1 carboxylate 


\section{Introduction}

Myriad detection strategies exist for chemical sensing. [1,2] Optical sensing is attractive because it can be minimally invasive and it allows stand-off detection. [1,3-5] However, optical sensing is not without its limitations. In most cases, one must operate under conditions where the source, sensor, and/or detector do not exhibit appreciable drift/decomposition over time and the sensor per se must respond reversibly to the target analytes. In situations where there are system instabilities, one often resorts to kinetic or ratiometric methods. [6-9] Kinetic methods are often implemented within fluorescence-based schemes by using the sensing reporter excited-state fluorescence lifetime $(\tau)$ as the analyte-dependent information carrier. [10-13] Because $\tau$ often occurs on a time scale that is orders of magnitude shorter in comparison to system fluctuations and $\tau$ is generally intensity independent, this approach is an intrinsically less bias-prone detection modality. The down side of this approach is that the detection system is often substantially more complex and costly. Ratiometric methods are commonly exploited when the sensor reporter chemistry exhibits spectroscopically distinct absorbance and/or emission spectral bands that change in an analyte-dependent manner. One then need only measure the analyte-dependent absorbance and/or intensity ratio at two wavelengths to yield an analyte-dependent response. Thus, in ratiometric methods, one needs to discriminate the absorbance/intensity at two wavelengths. There are essentially three strategies for accomplishing this: 1) a spectrometer coupled to an array detector (CCD, photodiode array); 2) slew scanning to rapidly "jump" a monochromator between two wavelengths using a platform with a single photodetector (e.g., photomultiplier tube); or, 3) a system having two discrete detection channels for monitoring at two wavelengths. Each of these platforms can be costly, complicated, and/or consume substantial 
electrical power (e.g., CCDs, photomultiplier tubes). There are certainly advantages to platforms that have no moving parts (e.g., gratings) and that operate at low power.

In this paper, we report a complementary metal oxide semiconductor integrated circuit (CMOS IC) with a buried double junction (BDJ) [14-16] photodiode combined with on-board signal processing circuitry that provides a direct measurement of the intensity ratio at two emission wavelengths and eliminates the need for any moving parts, external circuitry, or optical filters. Although BDJ platforms have been used previously to realize color-sensitive detection, [14-16] this strategy has been used just one other time in chemical sensing to minimize Rayleigh scatter and perform detection at a single emission wavelength. [17] Here, we report a BDJ platform for real-time ratiometric intensity measurements at two emission wavelengths coupled with dual wavelength ratiometric chemical sensors for detecting gaseous $\mathrm{NH}_{3}$ and determining aqueous solution $\mathrm{pH}$. The $\mathrm{NH}_{3}$-responsive sensor is based on a recently developed photonic ionic liquid consisting of trihexyl(tetradecyl)phosphonium $\left(\left[\mathrm{P}_{14,666}{ }^{+}\right]\right)$cations paired with 8-hydroxypyrene-1,3,6-trisulfonate (HPTS ${ }^{3-}$, pyranine). [18] The $\left[\mathrm{P}_{14,666}{ }^{+}\right]_{3}\left[\mathrm{HPTS}^{3-}\right]$ fluorescence emission spectrum exhibits two well-resolved bands that we previously used in a ratiometric mode for $\mathrm{NH}_{3}, \mathrm{MeNH}_{2}, \mathrm{EtNH}_{2}, n-\mathrm{PrNH}_{2}, \mathrm{Et}_{2} \mathrm{NH}$, and $\mathrm{Et}_{3} \mathrm{~N}$ detection by using slew scan fluorescence (SSF) with a conventional monochromator as the wavelength selection device and photomultiplier tube as the detector. The aqueous $\mathrm{pH}$ sensor is based on seminaphthorhodamine-1 carboxylate (SNARF-1) sequestered within a sol-gel-derived xerogel film. [19] SNARF-1 exhibits a fluorescence emission spectrum with two $\mathrm{pH}$-dependent fluorescence bands that have been exploited for ratiometric $\mathrm{pH}$ measurements in a variety of complex systems. [20,21]

\section{Materials and Methods}


2.1. Sensor Fabrication and Sample Preparation. $\left[\mathrm{P}_{14,666}{ }^{+}\right]_{3}\left[\mathrm{HPTS}^{3-}\right]$ was prepared as described elsewhere. [18] Thin films were formed by spin coating (500 RPM, $100 \mathrm{~s}) 250 \mu \mathrm{L}$ of a $10 \mathrm{mM}\left[\mathrm{P}_{14,666}{ }^{+}\right]_{3}\left[\mathrm{HPTS}^{3-}\right]$ solution (in EtOH) onto clean quartz microscope slides. SNARF-1 (Life Technologies) doped xerogels were prepared by using a protocol adapted from Dobmeier et al. [19] Briefly, a tetramethoxysilane (TMOS, Sigma-Aldrich) sol was prepared by mixing 1000 $\mu \mathrm{L}$ of doubly-distilled deionized $\mathrm{H}_{2} \mathrm{O}\left(\mathrm{ddH}_{2} \mathrm{O}\right), 500 \mu \mathrm{L}$ of TMOS, and $10 \mu \mathrm{L}$ of $0.04 \mathrm{M} \mathrm{HCl}$ (J.T. Baker) followed by 30 min sonication on ice. A SNARF-1-doped sol was prepared by mixing $75 \mu \mathrm{L}$ of the aforementioned TMOS sol, $32 \mu \mathrm{L}$ of 5 mM SNARF-1 dissolved in $\mathrm{ddH}_{2} \mathrm{O}$, and $300 \mu \mathrm{L}$ of $0.01 \mathrm{M} \mathrm{pH} 7.4$ phosphate buffer for $30 \mathrm{~s}$ using a touch mixer. A SNARF-1-doped xerogel film was formed on a clean borosilicate glass microscope slide by spin coating $200 \mu \mathrm{L}$ of the SNARF-1-doped sol for $30 \mathrm{~s}$ at 1000 RPM. Spin coating was repeated twice to create a thick and robust xerogel film. The xerogel films were dried in a vacuum oven at $100{ }^{\circ} \mathrm{C}$ for $1 \mathrm{~h}$.

Gaseous $\mathrm{NH}_{3}$ was prepared in situ within an Erlenmeyer flask by mixing $\mathrm{NH}_{4} \mathrm{Cl}$ (SigmaAldrich) with an excess of $2 \mathrm{M} \mathrm{NaOH}$ (Sigma-Aldrich) in $\mathrm{ddH}_{2} \mathrm{O}$. The $\mathrm{NH}_{3}$ vapor was directed from the flask into a flow cell//[ $\left.\mathrm{P}_{14,666}{ }^{+}\right]_{3}\left[\mathrm{HPTS}^{3-}\right]$ film assembly. [18] Raoult's law was used to calculate the gaseous analyte concentration within the flow cell. Aqueous $\mathrm{pH}$ solutions consisted of $10 \mathrm{mM}$ phosphate buffers between $\mathrm{pH} 6$ and 9 in $0.02 \mathrm{pH}$ increments. Solution $\mathrm{pH}$ values were determined with a calibrated glass $\mathrm{pH}$ electrode (Orion).

SSF measurements were recorded by measuring the $I_{511} / I_{445}$ or $I_{620} / I_{580}$ intensity ratio from the $\mathrm{NH}_{3}$ and $\mathrm{pH}$ sensors, respectively. There was typically no detectable hysteresis observed in either sensor platform.

2.2. BDJ Fabrication. Traditionally, image detectors that produce color-dependent responses use optical filters, such as Bayer filters, [22] requiring post-fabrication processing to 
attach the optical filters to the chip, which means that filter replacement is very difficult. Also, although optical filters can help to separate the excitation from the emission, they often attenuate the desired signal. Here, a BDJ platform is fabricated by CMOS technology in the ON Semi 0.5 $\mu \mathrm{m}$ process available through MOSIS (www.mosis.com). CMOS is particularly attractive because optoelectronic current generation associated with the optical sensor wavelengthdependent output and signal processing can be integrated within a single chip. In the current design, the BDJ photodiode active area is $78 \mu \mathrm{m} \times 78 \mu \mathrm{m}$.

2.3. BDJ Circuit and Operational Principles. Figure 1 presents a simplified schematic of a SSF system (A), a BDJ chip photograph (B), a BDJ structure schematic in side view (C), and the basic on-chip BDJ signal-processing topology (D). Figure 2 presents the detailed BDJ detector signal-processing circuit schematic (A) and a typical wavelength-dependent voltage output from the CMOS BDJ detector (B).

BDJ operation is based on depth and wavelength-dependent absorption through the silicon layer atop the detection elements [23] and is given by:

$$
I(z)=(1-R) I_{0} \exp (-a(\lambda) z)
$$

In this expression, $z$ is depth into the silicon layer, $R$ is the air-silicon interface reflection coefficient, $I_{0}$ is the incident light intensity, and $\alpha(\lambda)$ is the wavelength-dependent silicon absorption coefficient. The silicon $\alpha(\lambda)$ across the visible to near infrared spectral region decreases with increasing wavelength, so longer wavelengths penetrate deeper into the device before being absorbed by silicon. Analytically, this feature can be exploited to extract different wavelength signals from different depths within a BDJ device. [16] 
As shown in Figure 1C, we create three different silicon layers ( $p$-base, $n$-well and $p$ substrate) at different depths along the same vertical path $(z)$. Thus, there are two distinct depletion regions (one at each $p$ - $n$ junction), hence two locations for optoelectronic current generation. The two vertically stacked diodes enable simultaneous measurement of intensity and wavelength information based on Eqn. 1. Specifically, the current $\left(I_{i}\right)$ ratio from each $p-n$ junction produces a monotonic output with respect to wavelength. Further, by controlling the voltage on each $p-n$ junction, which changes the depletion region width in the $z$-direction, one can tune/optimize the output current for different wavelengths, providing additional control over the detector response. [16] This control can be exploited to finely select and/or tune wavelengths of interest without the need for any extrinsic optical filters or movable parts.

Figure 1D illustrates the BDJ detector topology. Briefly, the two input stages serve to clamp the two $p$ - $n$ junction bias voltages for a given BDJ pixel. In addition, by controlling the bias voltage through these stages, one can control the depletion region width in the $z$-direction for each diode, which can be used to tune the wavelength selectivity, as described previously. The output currents are mirrored to use for later stage calculations. The current from the common BDJ center node represents the total current through both photodiodes. Thus, a subtraction circuit removes $I_{1}$ from $I_{1}+I_{2}$ to determine the current through the lower $p$ - $n$ junction exclusively, $I_{2}$. To extend the device ability to operate over larger intensity ranges reaching the BDJ, we implement a logarithmic current-to-voltage converter circuit. The final stage is a differential amplifier that produces an output signal that is the difference of $V_{2}$ and $V_{1}$, which is the logarithmic value of $I_{2} / I_{1}$

Figure 2A shows the detailed BDJ circuit schematic. Figure 2B shows a typical wavelength-dependent BDJ response between 400 and $800 \mathrm{~nm}$ measured by using a broadband 
light source coupled to a wavelength-calibrated monochromator. For these particular experiments, the monochromator step size was $10 \mathrm{~nm}$, the spectral band pass was $0.2 \mathrm{~nm}$, and average beam fluence at the BDJ surface was $\sim 1 \mu \mathrm{W} \mathrm{mm}$. $^{-2}$.

2.4. Measurement Conditions. All experiments were performed at 293-295 K. The SSF experiments we carried out by using a photomultiplier tube-based spectrofluorimeter with excitation at $405 \mathrm{~nm}$ (or $532 \mathrm{~nm}$ ) and slewing scanning between 445 and $511 \mathrm{~nm}$ (or 580 and 620 $\mathrm{nm}$ ), respectively. Typical BDJ experiments were carried out with laser excitation at 405 or 532 $\mathrm{nm}(5-10 \mathrm{~mW})$. The CMOS BDJ detector chip was powered with a single 5V DC supply, and the total average chip power consumption was $<200 \mu \mathrm{W}$ during operation.

2.5. Statistics. Multiple data sets were acquired for each experiment and typical results are presented. Statistical significance was assessed by using one way ANOVA at the 95\% confidence level with pairwise comparison (Holm-Sidak test) $(p<0.05$ being significant). In all cases, the power of performance test exceeded 0.95 .

\section{Results and Discussion}

Figure 3 illustrates the $\left[\mathrm{P}_{14,666}{ }^{+}\right]_{3}\left[\mathrm{HPTS}^{3-}\right]$ chemical structure (A) and presents typical normalized steady-state emission spectra in air and in the presence of $4000 \mathrm{ppm} \mathrm{NH}_{3}$ (B) when excited at $405 \mathrm{~nm}$. In air, the emission consists of a single peak centered at $\sim 445 \mathrm{~nm}$ corresponding to emission from HPTS-OH (the species shown in Figure 3A). In the presence of $\mathrm{NH}_{3}$, a new peak grows in centered at $\sim 500 \mathrm{~nm}$, corresponding to emission from $\mathrm{HTPS}^{-} \mathrm{O}^{-}$and/or HTPS- $\mathrm{O}^{-} \cdots\left(\mathrm{NH}_{4}{ }^{+}, \mathrm{H}_{3} \mathrm{O}^{+}\right)$. As the $\mathrm{NH}_{3}$ concentration increases, the $I_{511} / I_{445}$ ratio increases. For direct reference, Figure 3B also shows the wavelength-dependent BDJ response (dashed profile) across the $\left[\mathrm{P}_{14,666}{ }^{+}\right]_{3}\left[\mathrm{HPTS}^{3-}\right]$ emission window. 
Figure 4 compares the $\mathrm{NH}_{3}$-dependent responses from a $\left[\mathrm{P}_{14,666}{ }^{+}\right]_{3}\left[\mathrm{HPTS}^{3-}\right]$-based sensor recorded using the SSF system $\left(I_{511} / I_{445}\right)$ (black) and the filter-free BDJ (red). The response profiles are very similar in light of the logarithmic value of $I_{2} / I_{1}$ from the BDJ platform. The $\mathrm{NH}_{3}$ detection limit using the BDJ is $0.1 \mathrm{ppm}$. As shown in the inset, the BDJ response time is $<1 \mathrm{~s}$, even with 1 meter of tubing between the sample and the sensor. The intrinsic BDJ response time is $\sim 0.05 \mathrm{~s}$.

Figure 5 illustrates the SNARF-1 chemical structure (A) and typical steady-state emission spectra for a SNARF-1-doped xerogel film sensor at pH 6.0 and 9.0 (B) when excited at $532 \mathrm{~nm}$. At $\mathrm{pH}$ 6.0, the SNARF-1 emission spectrum (B) is described by a single peak centered at $\sim 580$ $\mathrm{nm}$, corresponding to emission from protonated SNARF-1. As the $\mathrm{pH}$ is increased, the SNARF-1 deprotonates and a new peak/shoulder is observed in the emission spectrum at $\sim 620 \mathrm{~nm}$. The $I_{620} / I_{580}$ ratio increases as the solution $\mathrm{pH}$ increases. Figure $5 \mathrm{~B}$ also shows the wavelengthdependent BDJ response (dashed profile) over the SNARF-1 emission window. (Note: Although this window overlaps partially with the wavelength window shown in Figure 3B, the BDJ responses are different because the applied voltage on each $p-n$ junction is different and the depletion region width in the $z$-direction within the BDJ is slightly different in each experiment. The voltage differences are necessitated because of the differences in excitation and emission wavelengths.)

Figure 6 compares the $\mathrm{pH}$-dependent response from the SNARF-1-doped xerogel sensor excited at $532 \mathrm{~nm}$ using the SSF system $\left(I_{620} / I_{580}\right)$ (black) and the filter-free BDJ (red). The response profiles are again very similar in light of the logarithmic value of $I_{2} / I_{1}$ from the BDJ platform. The estimated $\mathrm{pH}$ resolution over the $\mathrm{pH} 6-9$ range is $0.03 \mathrm{pH}$ units. 
Finally, we explored the effects of source and/or sensor instability on the BDJ platform performance. Toward this end, we used a pH sensor, we modulated the excitation source intensity between 2 and $10 \mathrm{~mW}$ in a sinusoidal manner at 1, 10, 50 and $200 \mathrm{~Hz}$, and we measured the BDJ response and relative standard deviation (RSD) at $\mathrm{pH}$ 8.5. The average response and RSD were modulation frequency independent and also independent of BDJ sampling frequency between 0.05 and $300 \mathrm{~Hz}(p>0.59)$. Under the same conditions using SSF measurements, the average response differed by as much as 5-fold $(p<0.001)$ with a RSD exceeding 300\%, depending on the exact combination of slew scan frequency, source modulation frequency, and where in the source modulation cycle one initiated the SSF measurement. When the source power was randomly switched between 5 and $100 \mathrm{~mW}$ during a SSF experiment such that SNARF-1 became irreversibly photobleached at the higher laser power, the SSF-based response differed by up to 50 -fold near the switching time point, stabilizing thereafter until the next source power jump was implemented. With the BDJ, there was no detectable difference in response before, during, or after a source power jump ( $p>0.35$ ). The only time the BDJ failed to perform reliably or in any way acted inferior when compared to the SSF system was when the optical signals from the sensors were extremely weak $\left(<15 \mathrm{pW} \mathrm{mm}^{-2}\right)$.

\section{Conclusions}

We have reported a filter-free BDJ-based photodetector chip and shown that it is an excellent alternative to a slew scan fluorimeter coupled to a photomultiplier tube for ratiometric sensing applications. The commercially-fabricated BDJ chip is very small, lightweight $(<5 \mathrm{~g})$, consumes very little electrical power, and it is ideally suited for filter-free, multicolor photoluminescence detection.

\section{Acknowledgements}


This research is based in part upon work supported by the National Science Foundation under Grant Number CHE-0848171 and CHE-1411435 (to FVB). Any opinions, findings, and conclusions or recommendations expressed in this material are those of the authors and do not necessarily reflect the views of the National Science Foundation.

\section{Reference List}

[1] R.V. Harrison (Ed.), Chemical Sensors: Properties, Performance and Applications, Nova Science Publishers, Inc., 2010.

[2] J. Janata, R.J. Huber (Eds.), Solid State Chemical Sensors, Academic Press, Inc., 1985.

[3] V.M. Mirsky, A.K. Yatsimirsky (Eds.), Artificial Receptors For Chemical Sensors, WileyVCH Verlag GmbH \& Co. KGaA, 2010.

[4] O.S. Wolfbeis (Ed.), Electrochemical and Optical Chemical Sensors [In: Mikrochim. Acta. 1995; 121(1-4)], Springer, 1995.

[5] O.S. Wolfbeis (Ed.), Fiber Optic Chemical Sensors and Biosensors, CRC, 1991.

[6] H.A. Mottola, Kinetic Aspects of Analytical Chemistry, John Wiley \& Sons, 1988.

[7] A.P. Demchenko, The future of fluorescence sensor arrays, Trends Biotechnol., 23 (2005) 456-460.

[8] P. Carol, S. Sreejith, A. Ajayaghosh, Ratiometric and near-infrared molecular probes for the detection and imaging of zinc ions, Chem. Asian J., 2 (2007) 338-348.

[9] M. Schäferling, A. Duerkop, Intrinsically referenced fluorimetric sensing and detection schemes: Methods, advantages and applicationsin, U. Resch-Genger (Ed.) (Springer 
Series on Fluorescence), Springer Berlin Heidelberg, 2008, pp. 373-414.

[10] H. Szmacinski, J.R. Lakowicz, Fluorescence lifetime-based sensing and imaging, Sens. Act., B29 (1995) 16-24.

[11] A.G. Ryder, S. Power, T.J. Glynn, Evaluation of acridine in Nafion as a fluorescence lifetime based pH sensor, Appl. Spectrosc., 57 (2003) 73-79.

[12] C. Baleiza, S. Nagl, M. Schaferling, M.N. Berberan-Santos, O.S. Wolfbeis, Anal. Chem. 80 (2008) 6449-6457.

[13] R. Esposito, B.D. Ventura, S. De Nicola, C. Altucci, R. Velotta, D.G. Mita, M. Lepore, Glucose sensing by time-resolved fluorescence of sol-gel immobilized glucose oxidase, Sensors, 11 (2011) 3483-3497.

[14] R.F. Wolffenbuttel, P.P.L. Regtien, “A novel approach to solid-state color sensing, Sens. Actuators, 9 (1986) 199-211.

[15] O. Hofmann, X. Wang, A. Cornwell, S. Beecher, A. Raja, D.D.C. Bradley, A.J. deMello, J.C. deMello, Monolithically integrated dye-doped PDMS long-pass filters for disposable on-chip fluorescence detection, Lab Chip, 6 (2006) 981-987.

[16] S. Feruglio, G.-N. Lu, P. Garda, G. Vasilescu, A review of the CMOS buried double junction (BDJ) photodetector and its applications, Sensors, 8 (2008) 6566-6594.

[17] Z. Zhan, B. Zhou, Z. Fu, F.V. Bright, A.N. Cartwright, C.M. Bartsch, A.H. Titus, Filterless optical oxygen sensor based on a CMOS buried double junction photodiode, Sens. Actu. B, 176 (2013) 729-735. 
[18] K.Y. Yung, A.J. Schadock-Hewitt, N.P. Hunter, F.V. Bright, G.A. Baker, 'Liquid litmus': Chemosensory pH-responsive photonic ionic liquids, Chem. Commun., 47 (2011) 47754777.

[19] K.P. Dobmeier, G.W. Charville, M.H. Schoenfisch, Nitric oxide-releasing xerogel-based fiber-optic pH sensors, Anal. Chem., 78 (2006), 7461-7466.

[20] P.E.J. Van Erp, M.J.J.M. Jansen, G.J. De Jongh, J.B.M. Boezeman, J. Schalkwijk, Ratiometric measurement of intracellular $\mathrm{pH}$ in cultured human keratinocytes using carboxy-SNARF-1 and flow cytometry, Cytometry, 12 (1991) 127-132.

[21] B.K. Grillo-Hill, B.A. Webb, D.L. Barber, Chapter 23 - Ratiometric imaging of pH probes,eth. Cell Biol., 123 (2014) 429-448.

[22] L. Shen, M. Ratterman, D. Klotzkin, I. Papautsky, A CMOS optical detection system for point-of-use luminescent oxygen sensing, Sens. Actuators, B, 155 (2011) 430-435.

[23] P. Würfel, T. Trupke, M. Rüduiger, T. Puzzer, E. Schäffer, W. Warta, S.W. Glunz, Diffusion lengths of silicon solar cells from luminescence images, J. Appl. Phys., 101 (2007) 123110-123120. 


\section{Figure Captions}

Figure 1. Measurement systems used in this research. (Panel A) Slew scan spectrofluorimeter. (Panel B) BDJ detector chip. (Panel C) Cross sectional view through a BDJ detector chip structure. (Panel D) Basic BDJ detector chip signal processing topology.

Figure 2. BDJ detector chip circuit and response profile. (Panel A) Detailed BDJ detector chip circuit diagram. (Panel B) Typical wavelength-dependent voltage output from a CMOS BDJ detector chip.

Figure 3. Ionic liquid chemical structure, emission spectra, and BDJ detector chip response. (Panel A) $\left[\mathrm{P}_{14,666}{ }^{+}\right]_{3}\left[\operatorname{HPTS}^{3-}\right]$ chemical structure. (Panel B) Typical normalized steady-state emission spectra $\left(\lambda_{\text {ex }}=405 \mathrm{~nm}\right)$ in air and $\mathrm{NH}_{3}$ vapor and wavelength-dependent BDJ detector chip response.

Figure 4. Typical $\mathrm{NH}_{3}$-dependent response from a $\left[\mathrm{P}_{14,666}{ }^{+}\right]_{3}\left[\mathrm{HPTS}^{3}\right]$ thin film sensor recorded using the slew scan fluorimeter (black) and the filter-free BDJ CMOS IC (red). (Inset) Timedependent response profile to a $\mathrm{NH}_{3}$ pulse.

Figure 5. SNARF-1 chemical structure, emission spectra, and BDJ detectoe chip response. (Panel A) SNARF-1 chemical structure. (Panel B) Typical steady-state emission spectra $\left(\lambda_{\mathrm{ex}}=\right.$ $532 \mathrm{~nm})$ at $\mathrm{pH} 6.0$ and 9.0 and wavelength-dependent BDJ detector chip response. 
Figure 6. Typical pH-dependent response from a SNARF-1-doped xerogel thin film sensor recorded using the slew scan fluorimeter (black) and the filter-free BDJ CMOS IC (red). 


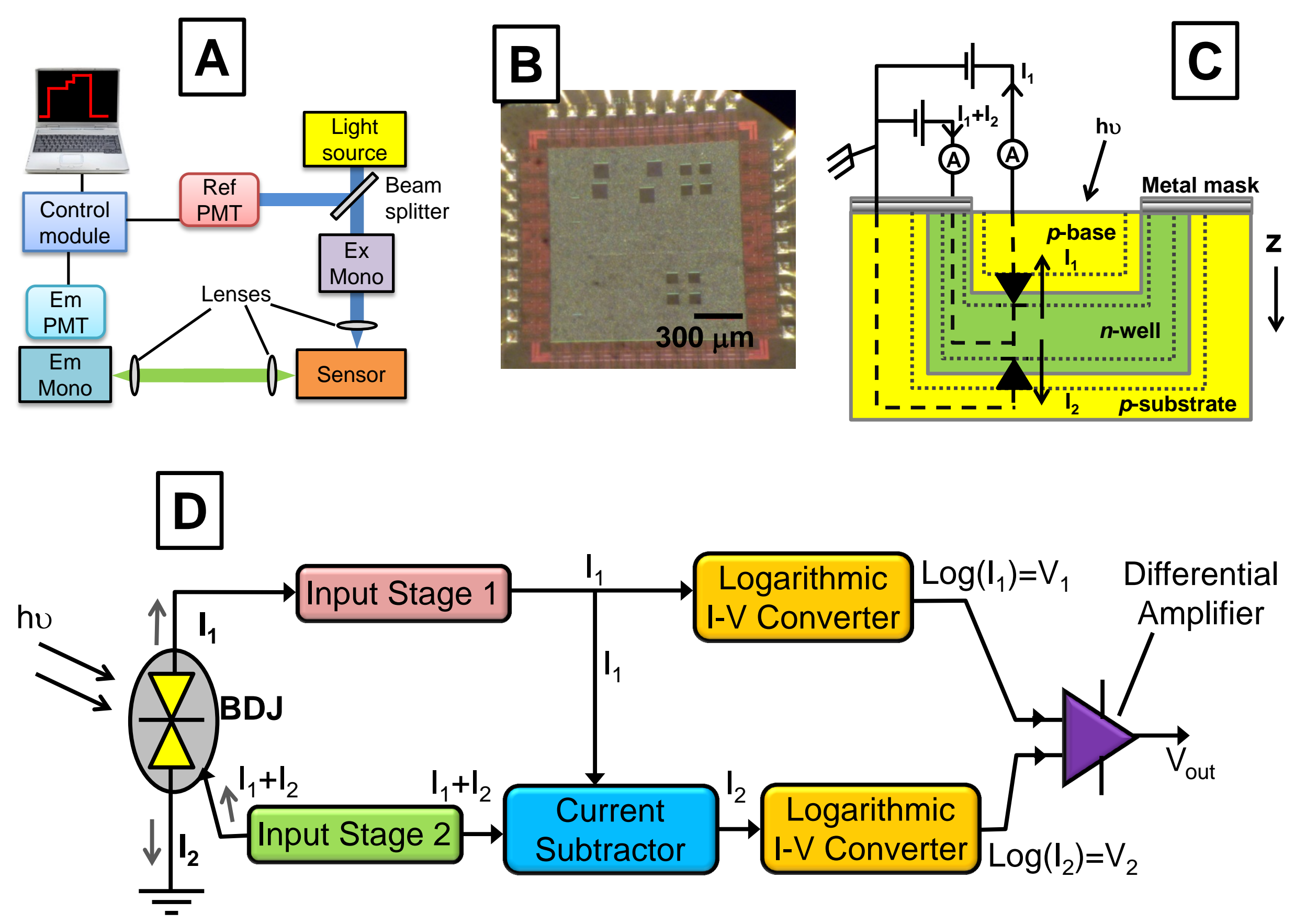




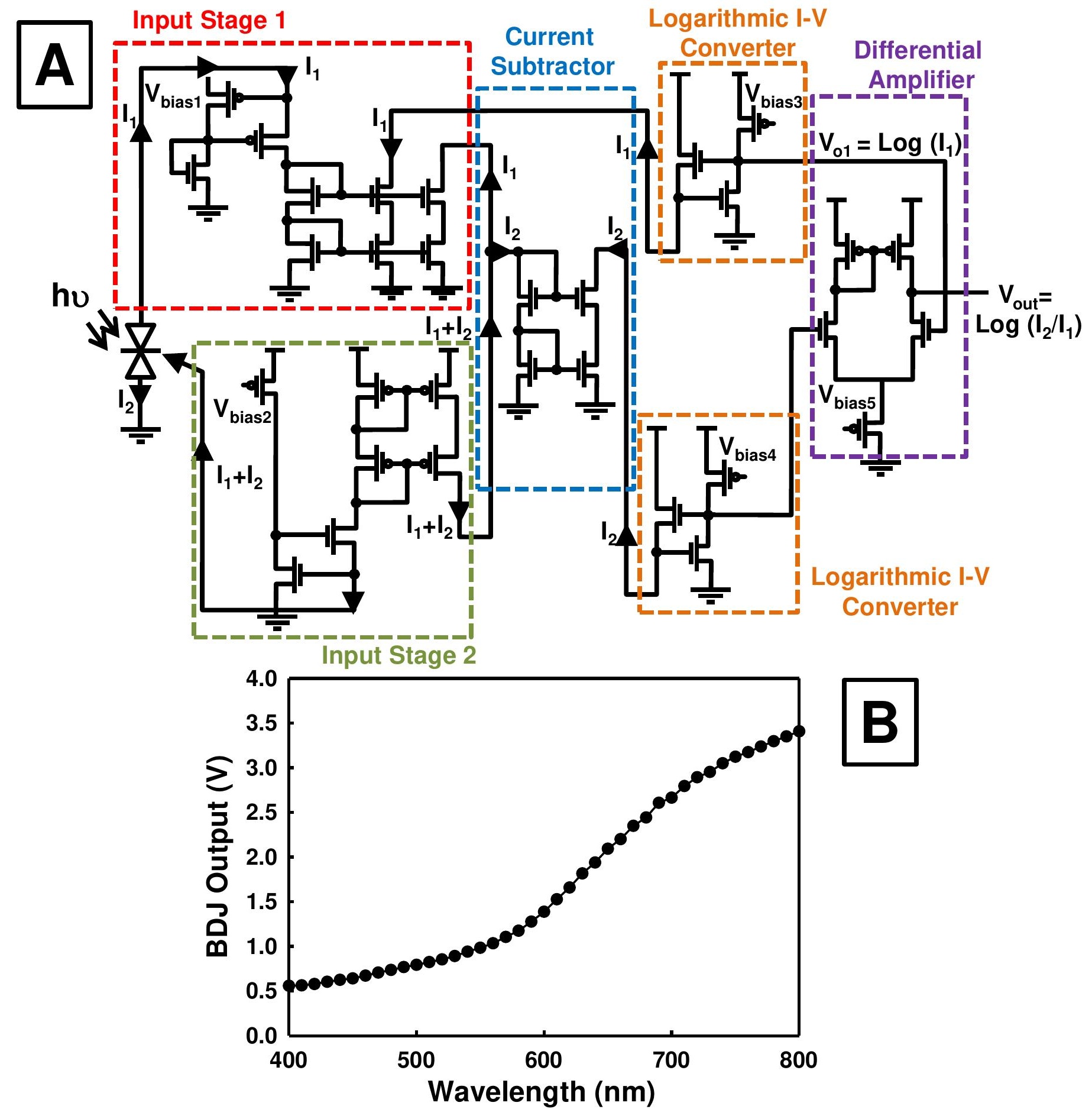

Figure 2 


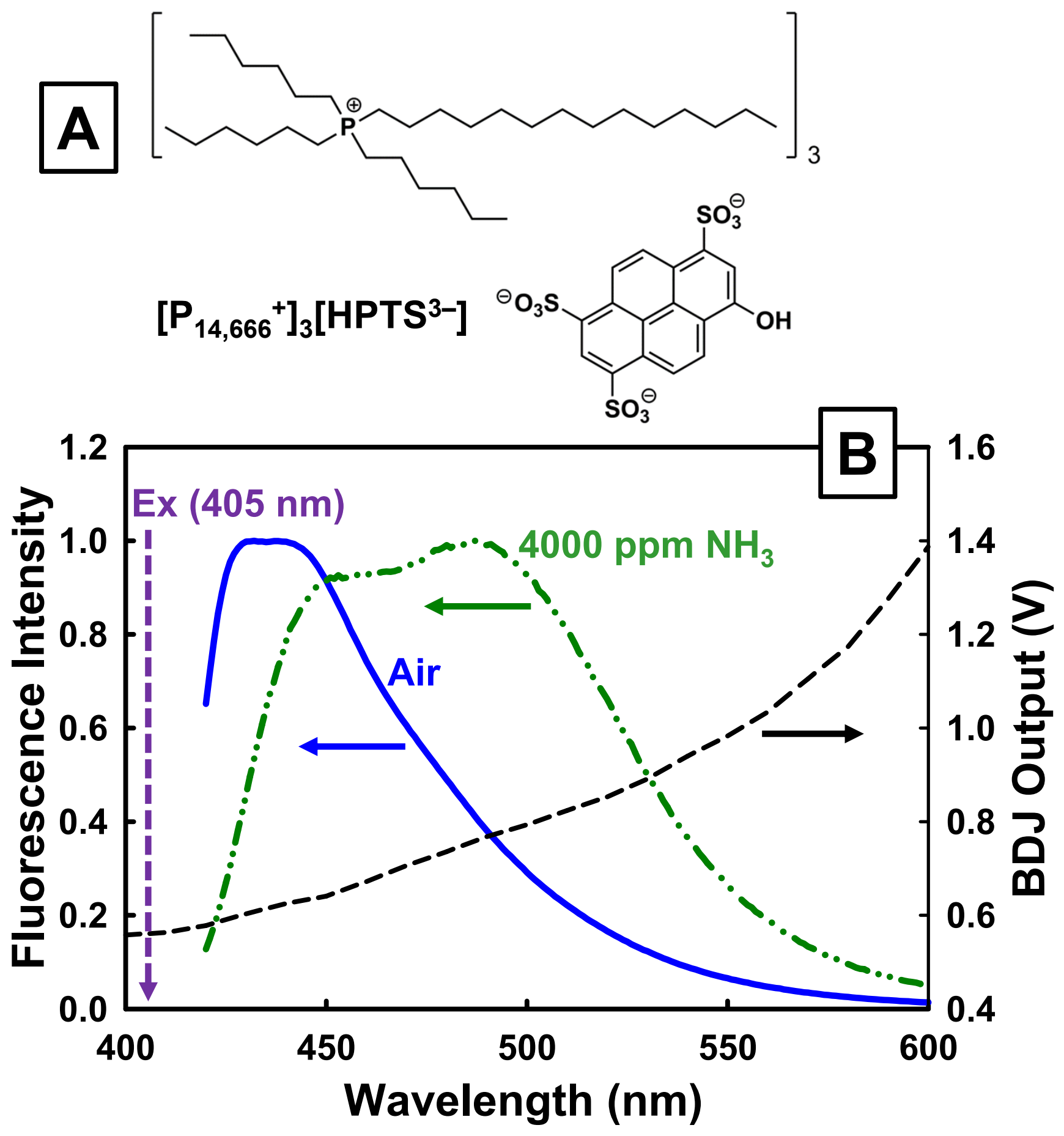

Figure 3 


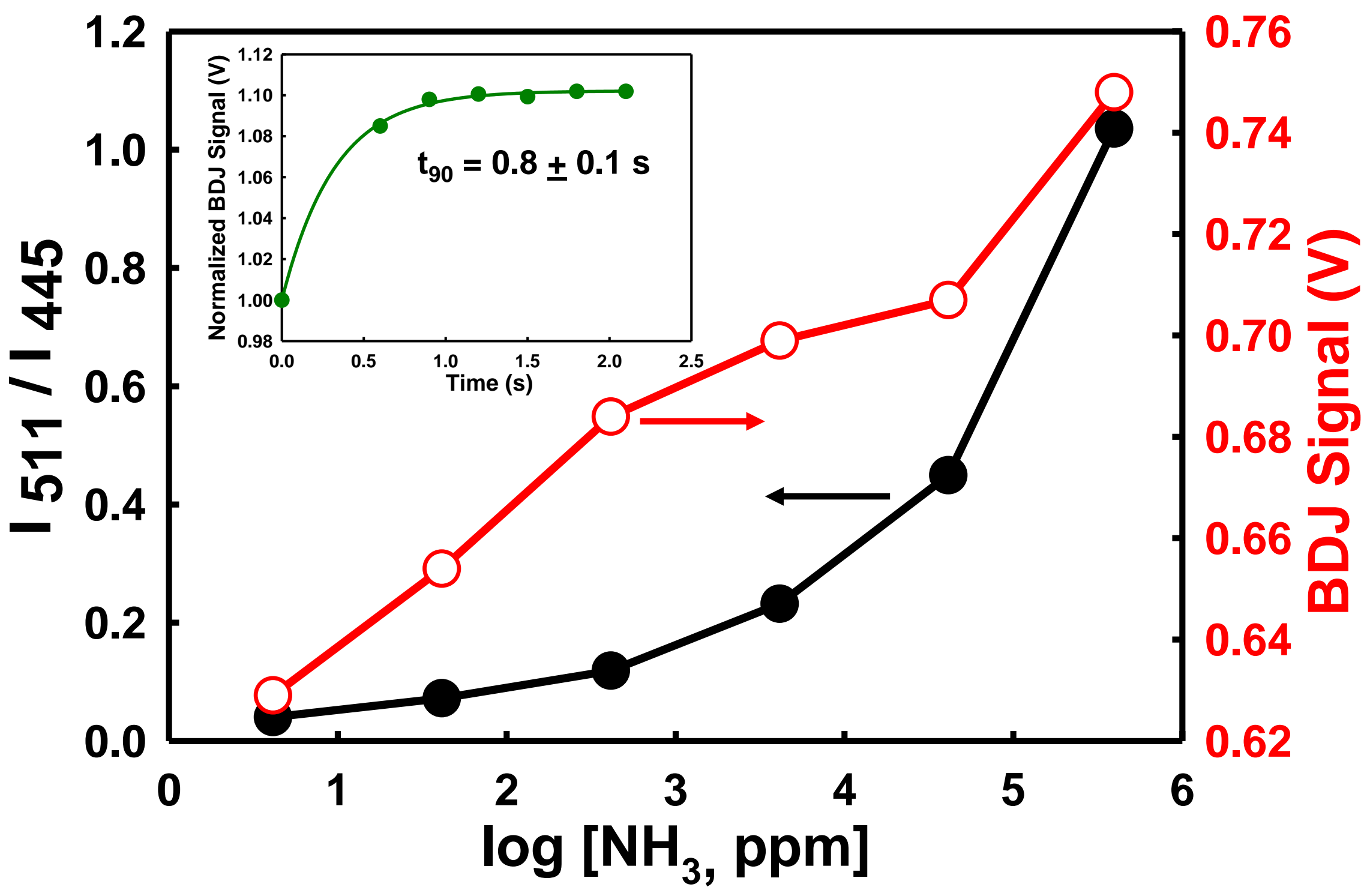

Figure 4 


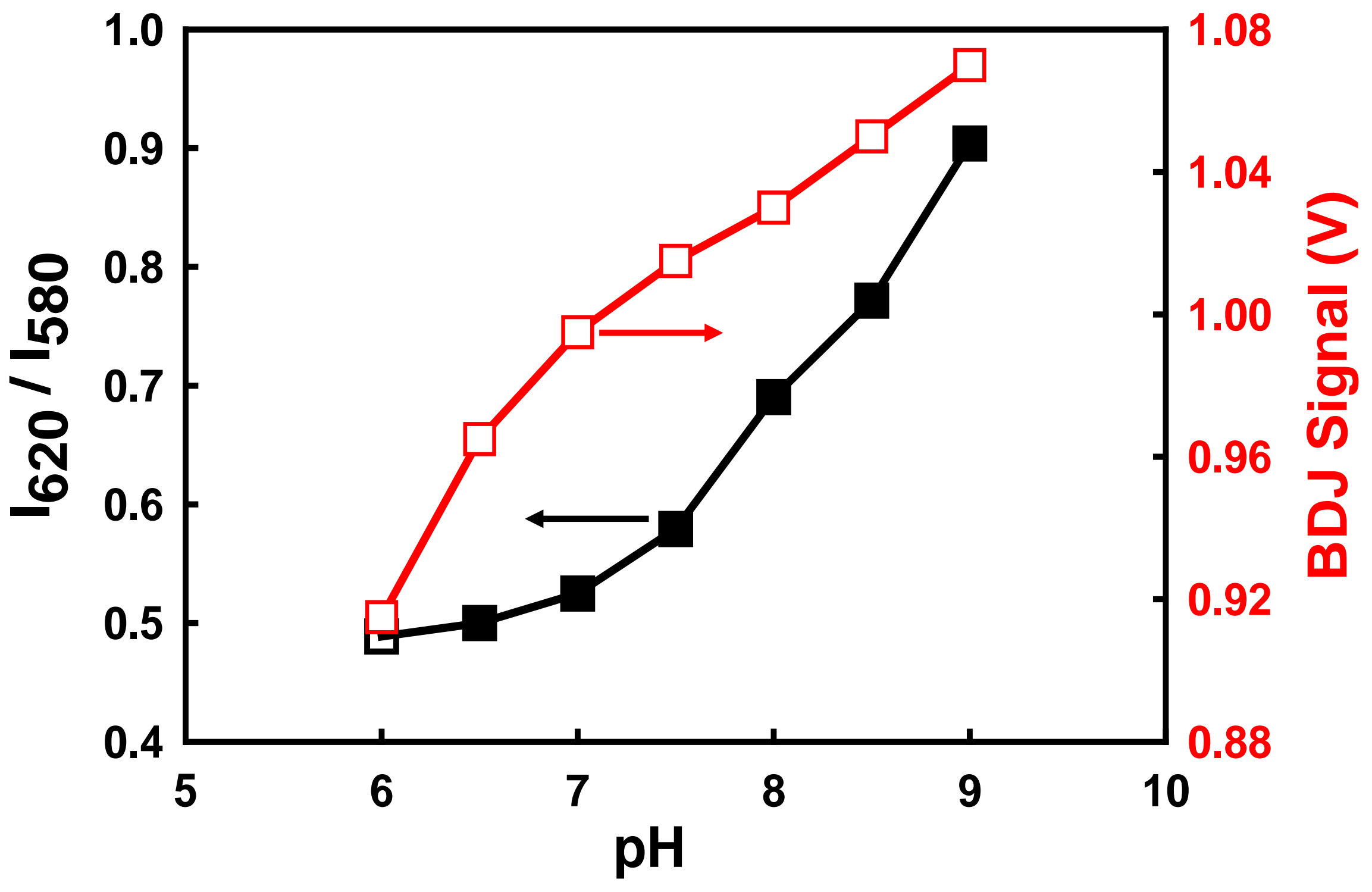

Figure 5 


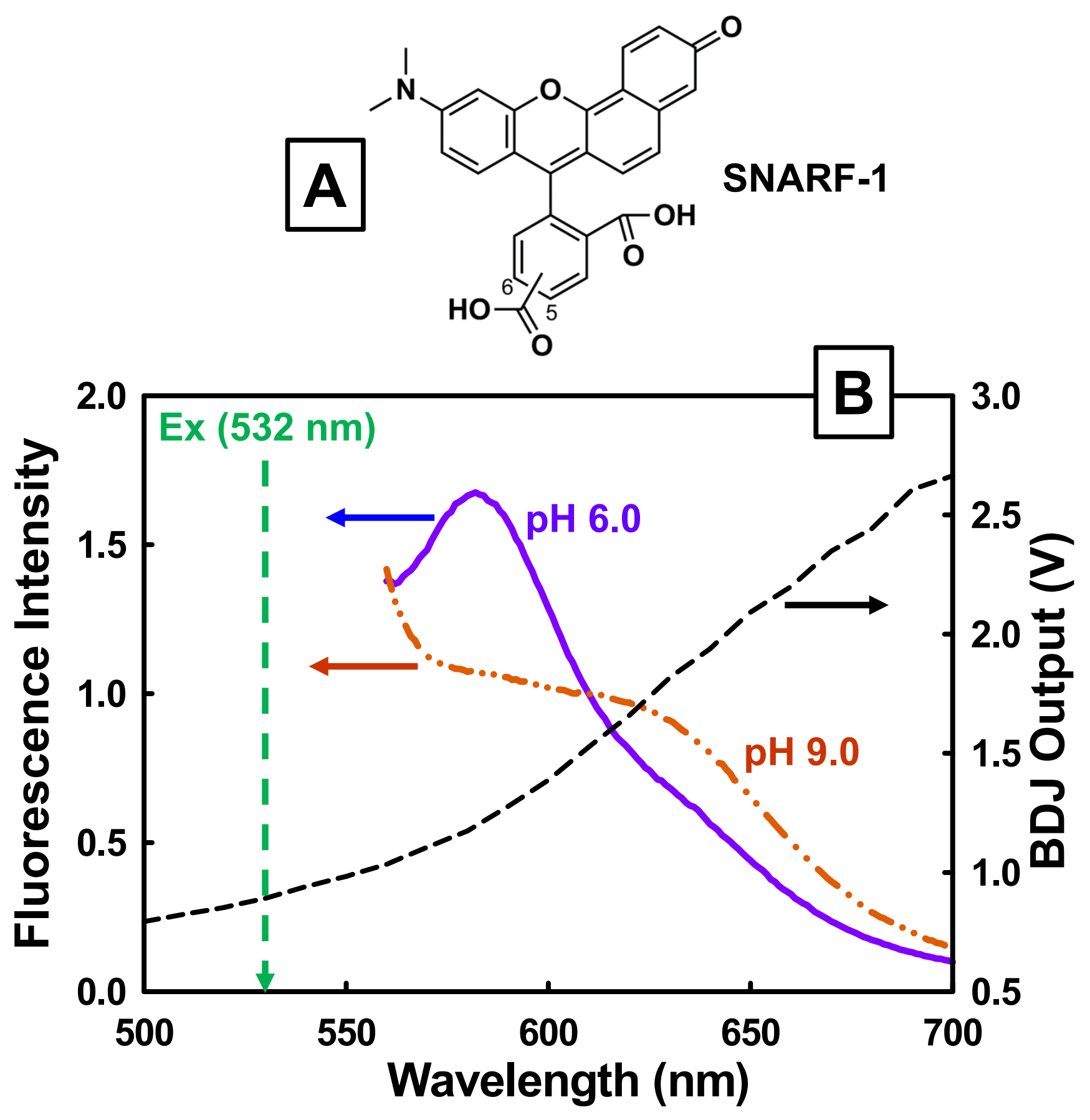

Figure 6 
Sensor

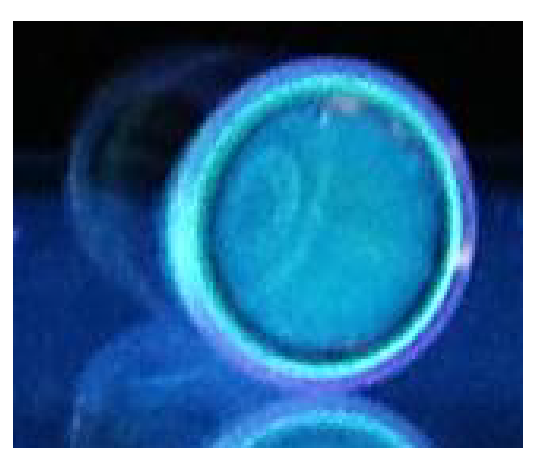

Responses

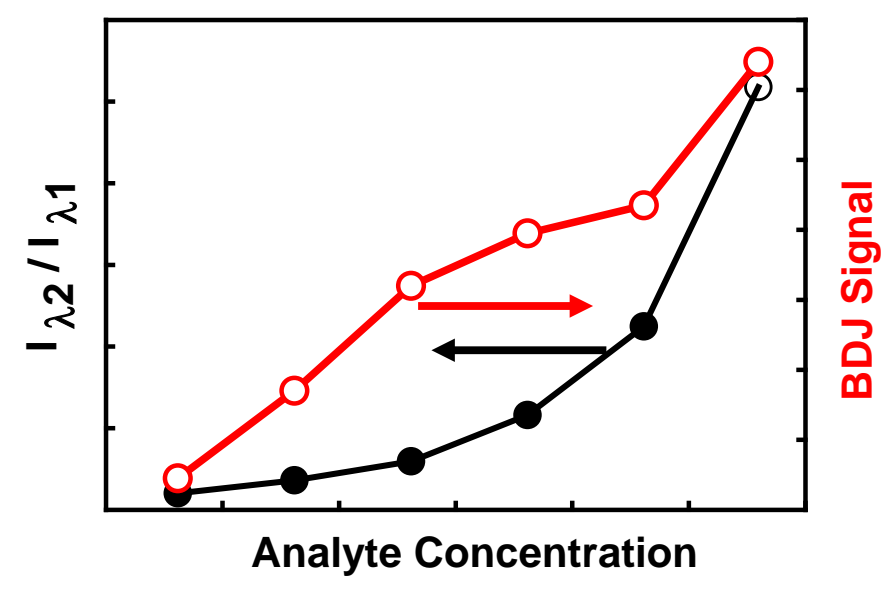

Spectrum
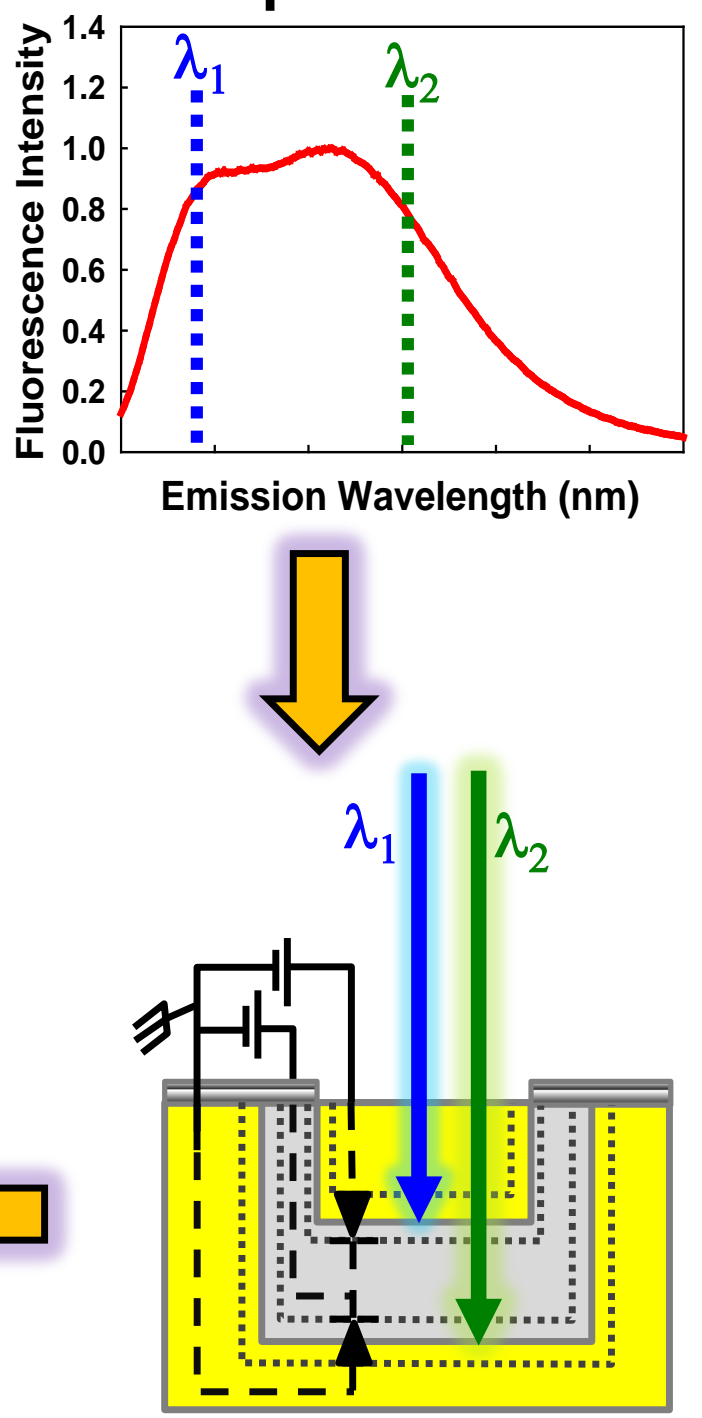

CMOS BDJ 